The Journal of Animal \& Plant Sciences, 31(2): 2021, Page: 416-422

ISSN (print): 1018-7081; ISSN (online): 2309-8694

\title{
COMPARATIVE EVALUATION OF BODY CONFORMATION TRAITS IN NATIVE ASEEL CHICKEN FED UNDER DIFFERENT DIETARY LYSINE REGIMENS
}

\author{
T. Batool ${ }^{1},{ }^{*}$ S. Farooq ${ }^{1}$, N. Roohi ${ }^{1}$ and A. Mahmud ${ }^{2}$ \\ ${ }^{1}$ Physiology/Endocrinology laboratory, Department of Zoology, University of the Punjab, Lahore, Pakistan \\ ${ }^{2}$ Department of Poultry Production, University of Veterinary and Animal Sciences, Lahore, Pakistan \\ Corresponding Author E-mail: tahirabatool1111@gmail.com
}

\begin{abstract}
An experiment was conducted for comparative evaluation of body conformation traits in Lakha, Mianwali, Mushki and Peshawari varieties of indigenous Aseel fed under three lysine regimens (L1, L2 and L3). In L1, $1.3 \%$ lysine offered from week 1-6 (one phase), in L2, 1.4 and 1.2\% lysine from week 1-3 and 4-6 (two phases), while 1.5, 1.3 and $1.1 \%$ lysine was offered from week 1-2, 3-4 and 5-6 (three phases), respectively in L3. Day-old-chicks ( $\mathrm{n}=240)$ including 60 per variety with 20 birds per treatment group were used in this study. Body weight, shank length, drumstick length, drumstick circumference, body length, keel length, wing span, wing spread and breast width were measured on weekly basis. The data after analysis of homogeneity and uniformity, were exposed to factorial ANOVA as a Randomized Complete Block Design. The results indicated that birds fed under lysine regimen L3 had significantly $(\mathrm{P} \leq 0.05)$ greater body weight, breast width, shank length, shank circumference, drumstick length, drumstick circumference, keel length as well as wing span and wing spread. Among Aseel varieties, Lakha and Mushki varieties showed more developed body conformation traits than other varieties and these birds may perform better in terms of their morphometrics when reared under lysine regimen L3.
\end{abstract}

Key words: Aseel varieties, Body weight, Conformation traits, Lysine regimens, Wing spread and Span.

https://doi.org/10.36899/JAPS.2021.2.0230

Published online October 03,2020

\section{INTRODUCTION}

Growth of organisms is a very complex phenomenon and is controlled through many intrinsic and extrinsic factors. Body mass and different body conformation traits are important to determine growth in native chicken. The mechanism of growth and its control is very complicated and not to be explained by univariate analysis due to the biological correlation of all these interrelated traits under the pleiotropic impact of genes and genetic linkage of loci (Rosario et al., 2008; Yunusa and Adeoti, 2014). Measurements of physical dimensions of alive birds can well predict the body size and its solidity i.e., breast angle or either breast depth and shank circumference. The evaluation of body size and conformation of particular species should always be the first approach in livestock description, while production performance and its improvement is an interlinked phenomenon being secondary to morphometric analysis (Chineke et al., 2002). Studies have been carried out showing the interrelationship of body weight and other morphogenetic characters (Ogah et al., 2011). This phenotypic correlation among various body conformation traits is due to both genotypic and environmental correlations among them, particularly when it is positive could be very helpful for selection of better breeds and strains (Yakubu and Mohammed, 2012).The body conformation traits can also determine the percent meat yield of carcass (Das et al., 2014) and are helpful for poultry farmers, exclusively the geneticists in purifying the market value of meat type bird's stock (Hussain et al., 2015). A comparative evaluation of body measurements could be of paramount importance for planning and improving effective conservation, breeding and economics of native breeds (Paleja et al., 2008).

The chicken of local origin like Aseel of IndoPak has a great historic importance, being ancestor of certain breeds like Cornish, the ancestor of male line of today's broiler (Dohner, 2001). Aseel has highest ultimate weight, solid physique, endurance and resistivity than any other native breed (Singh, 2001). In Pakistan, the indigenous Aseel chicken has many varieties but the most aggressive among them is Mushki, commonly called as "Siyah" with greenish black plumage, white eyes and shanks, red wattles and ear lobes. Its mature male and female have body weight of 2570 (gm) and 1870 (gm), respectively (Ahmad et al., 2014). Lakha variety is famous with common name "Cheena" having white dots on the whole plumage (mottled). The mature male and female has body weight in the range of 3000 3800 (gm) and 2500-3200 (gm), respectively (Babar et al., 2012). Peshaweri variety, also known as "Peela" due to its wheaten colored plumage and is very popular in Pakistan. It has red ear lobes, pea comb and yellow shanks while wattles are absent. The adult male usually attains body weight up to 2500 (gm), whereas, female 
attains 1700(gm) at sexual maturity (Sohail et al., 2013). Similarly, Mianwali variety being particular in its phenotypic characteristics from the other varieties, having dark brown coloration of plumage. The mature Mianwali male and female have body weight of 25003000 and 2000-2200 (gm), respectively (Babar et al., 2012). A substantial work has been accomplished recently with respect to growth (Hussain et al., 2018; Saima et al., 2010; Zia et al., 2017), physiology (Batool et al., 2017a), behavior (Batool et al., 2017b; Rehman et al., 2018), and production (Batool et al., 2018) of Pakistani indigenous Aseel chicken. However, scarce published work and information is available regarding its body conformation traits. The present study has been designed to evaluate the effect of different dietary lysine regimens on body conformation traits in four varieties of indigenous Aseel chicken with the objective that Aseel varieties may impose the feeding influence differently under various lysine regimens on their body conformations.

\section{MATERIALS AND METHODS}

The study was carried out between December 2014 to January 2015 for a period of six weeks at Indigenous Chicken Genetic Resource Center, University of Veterinary and Animal Sciences (UVAS), Lahore. The care and welfare of experimental birds was prioritized and institutional ethical committee's guidelines and protocol was strictly followed throughout the experiment. Day old Aseel chicks ( $n=240$, with equal no. of birds from both sexes and an average weight of $34 \mathrm{gm}$ ) including 60 numbers of each variety (Peshawari, Mushki, Mianwali and Lakha) were randomly selected from newly hatched stock at Avian Research and Training Center, UVAS, Lahore. These Aseel birds were sub-divided into three groups A, B and C. The birds were tagged as per their variety and feeding regimen with factorial arrangement of $3 \times 4 \times 20$ [(lysine regimens $\times$ Aseel varieties $\times$ replicates (with one bird in each to avoid fighting among Aseel birds)]. The birds were kept in three-tiered battery cage units with standard stocking density as was approved by institutional ethical committee for the welfare and well-being of experimental birds, equipped with nipple water drinkers for ad libitum water supply as well as removable feeders and dropping trays. These cages $(n=3)$ were placed in a well illuminated poultry house $(20 \times 20$ sq. ft. $)$ whose temperature range and relative humidity was maintained between $21-32^{\circ} \mathrm{C}$ and $60-75 \%$, respectively. The experimental birds were fed with three different dietary lysine regimens L1, L2 and L3, respectively for initial six weeks of their age. L1 lysine regimen containing $1.3 \%$ lysine was offered for six weeks to the birds of group A, $1.4 \%$ and $1.2 \%$ (L2) each for three weeks to group B and $1.5,1.3$, and $1.1 \%$ lysine (L3), each for subsequent two weeks to the birds of group C. Table 1 and 2 represents the chemical composition of experimental diet. Body weight (BW), shank length (SL), shank circumference (SC), drumstick length (DL), drumstick circumference (DC), body length (BL), keel bone length (KL), wing span (WSN), wing spread (WSD) and breast width (BWD) were measured for each selected bird per week according to the method as adopted by Udeh et al. (2011). Body weight was measured through digital weighing balance having capacity of weighing up to $5 \mathrm{gm}$ and shank length, drumstick length, body length, keel bone length, wing span, wing spread were measured by measuring scale with a minimum limit to measure up to $0.05 \mathrm{~mm}$. Vernier's caliper was used to measure shank, drumstick circumferences and breast width.

The collected data after examination of homogeneity and equality, were analyzed by using Statistical Analysis System (SAS Institute Inc., 2002-03, version 9.1.3) software, using two-way Analysis of Variance (ANOVA) as statistical tool and General Linear Model (GLM) procedures. Aseel birds were placed under blocks, while randomized complete block design (RCBD) was adopted as experimental design having dietary lysine regimens, Aseel varieties and interactions among them as principal effects. Treatment means were compared through Duncan's Multiple Range test and considered as significant at $\mathrm{P} \leq 0.05$.

\section{RESULTS}

Significant $(\mathrm{P} \leq 0.05)$ variations were found among different lysine regimens and varieties with respect to $\mathrm{BW}, \mathrm{SL}$ and $\mathrm{DL}$ and birds fed on L3 lysine regimen had highest BW $(409.59 \pm 4.62 \mathrm{~g})$ and developed largest SL $(4.58 \pm 0.06 \mathrm{~cm})$ and $\mathrm{DL}(5.78 \pm 0.06 \mathrm{~cm})$ followed by L2 with $383.05 \pm 4.39 \mathrm{~g}$, L2 $4.37 \pm 0.08$, $5.57 \pm 0.08 \mathrm{~cm}$ and L1 with $366.06 \pm 3.95 \mathrm{~g}, 4.17 \pm 0.62$, $5.27 \pm 0.06 \mathrm{~cm}$, respectively. Among varieties, Mushki had higher BW, while Lakha had higher SL and DL than other varieties (Table 3$)$. Significant $(P \leq 0.05)$ variations were also found in SC and DC, wherein, both L3 and L2 had greater SC $(2.32 \pm 0.03,2.32 \pm 0.03 \mathrm{~cm})$ and $\mathrm{DC}$ $(3.61 \pm 0.03,3.60 \pm 0.03 \mathrm{~cm})$ than L1 (both L3 and L2 were statistically non-significant among themselves). Among varieties, all except Peshawari had greater SC and DC (Table 3). The current study also revealed KL and BWD significantly $(P \leq 0.05)$ largest in birds fed on L3 $(5.18 \pm 0.06,1.45 \pm 0.03 \mathrm{~cm})$ followed by L2 $(4.97 \pm 0.08$, $1.29 \pm 0.02 \mathrm{~cm})$ and $\mathrm{L} 1(4.77 \pm 0.06,1.13 \pm 0.02 \mathrm{~cm})$ lysine regimen. KL of Lakha was larger than other varieties, while BWD of all three varieties were comparatively larger than Peshawari (Table 4). The birds fed on L3 had greater WSN and WSD i.e., $37.92 \pm 0.15$ and $18.71 \pm 0.15 \mathrm{~cm}$ than those fed on L2 and L1 lysine reg imens, while Lakha had largest WSD than Mushki, Mianwali and Peshawari varieties (Table 4). 
Table 1. Chemical composition of experimental diets.

\begin{tabular}{|c|c|c|c|c|c|}
\hline \multirow{2}{*}{ Ingredients } & \multicolumn{5}{|c|}{ Dietary lysine levels (\%) } \\
\hline & 1.1 & 1.2 & 1.3 & 1.4 & 1.5 \\
\hline Corn & 59.08 & 59.08 & 59.08 & 59.08 & 59.08 \\
\hline Sunflower Meal (24\%) & 18.90 & 18.90 & 18.90 & 18.90 & 18.90 \\
\hline Soya bean Meal (44\%) & 7.04 & 7.04 & 7.04 & 7.04 & 7.04 \\
\hline Rapeseed Meal (36\%) & 3.00 & 3.00 & 3.00 & 3.00 & 3.00 \\
\hline Fish Meal $(52 \%)$ & 3.00 & 3.00 & 3.00 & 3.00 & 3.00 \\
\hline Poultry by-product Meal (50\%) & 3.00 & 3.00 & 3.00 & 3.00 & 3.00 \\
\hline Molasses & 3.00 & 3.00 & 3.00 & 3.00 & 3.00 \\
\hline Limestone & 1.14 & 1.14 & 1.14 & 1.14 & 1.14 \\
\hline Lysine Sulphate & 0.75 & 0.80 & 0.90 & 1.00 & 1.10 \\
\hline Mono Calcium Phosphate & 0.45 & 0.45 & 0.45 & 0.45 & 0.45 \\
\hline Vitamin-Mineral Premix* & 0.20 & 0.20 & 0.20 & 0.20 & 0.20 \\
\hline Sodium Chloride & 0.18 & 0.18 & 0.18 & 0.18 & 0.18 \\
\hline Alimet (Novus) & 0.17 & 0.17 & 0.17 & 0.17 & 0.17 \\
\hline Betaine $\mathrm{HCl}$ & 0.05 & 0.05 & 0.05 & 0.05 & 0.05 \\
\hline Threonine & 0.04 & 0.04 & 0.04 & 0.04 & 0.04 \\
\hline
\end{tabular}

Table 2. Calculated nutritional composition of experimental diets.

\begin{tabular}{lccccc}
\hline \multirow{2}{*}{ Nutrients (\%) } & \multicolumn{5}{c}{ Dietary lysine level \% } \\
\cline { 2 - 6 } & $\mathbf{1 . 1}$ & $\mathbf{1 . 2}$ & $\mathbf{1 . 3}$ & $\mathbf{1 . 4}$ & $\mathbf{1 . 5}$ \\
\hline Metabolize Energy (Kcal/kg) & 2746.99 & 2753.69 & 2760.39 & 2767.09 & 2773.79 \\
Dry Matter & 87.17 & 87.36 & 87.56 & 87.76 & 87.96 \\
Crude Protein & 17.06 & 17.18 & 17.29 & 17.40 & 17.51 \\
Crude Fiber & 6.93 & 6.93 & 6.93 & 6.93 & 6.93 \\
Ash & 4.09 & 4.09 & 4.09 & 4.09 & 4.09 \\
Either Extract & 3.59 & 3.59 & 3.59 & 3.59 & 3.59 \\
Calcium & 0.84 & 0.84 & 0.84 & 0.84 & 0.84 \\
Chloride & 0.22 & 0.22 & 0.22 & 0.22 & 0.22 \\
Sodium & 0.16 & 0.16 & 0.16 & 0.16 & 0.16 \\
Total phosphorus & 0.68 & 0.68 & 0.68 & 0.68 & 0.68 \\
Potassium & 0.71 & 0.71 & 0.71 & 0.71 & 0.71 \\
Digestible phosphorus & 0.36 & 0.36 & 0.36 & 0.36 & 0.36 \\
Linoleic Acid & 1.42 & 1.42 & 1.42 & 1.42 & 1.42 \\
Lysine & 1.1 & 1.2 & 1.3 & 1.4 & 1.5 \\
Methionine & 0.45 & 0.45 & 0.45 & 0.45 & 0.45 \\
Methionine + Cysteine & 0.78 & 0.78 & 0.78 & 0.78 & 0.78 \\
Digestible Arginine & 0.98 & 0.98 & 0.98 & 0.98 & 0.98 \\
Digestible Tryptophan & 0.14 & 0.14 & 0.14 & 0.14 & 0.14 \\
Digestible Threonine & 0.57 & 0.57 & 0.57 & 0.57 & 0.57 \\
Digestible Lysine & 0.99 & 1.09 & 1.20 & 1.31 & 1.41 \\
Digestible methionine & 0.42 & 0.42 & 0.42 & 0.42 & 0.42 \\
Digestible Methionine + Cysteine & 0.67 & 0.67 & 0.67 & 0.67 & 0.67 \\
Threonine & 0.67 & 0.67 & 0.67 & 0.67 & 0.67 \\
Tryptophan & 0.19 & 0.19 & 0.19 & 0.19 & 0.19 \\
Arginine & 1.10 & 1.10 & 1.10 & 1.10 & 1.10 \\
Cysteine & 0.32 & 0.32 & 0.32 & 0.32 & 0.32 \\
Digestible Cysteine & 0.26 & 0.26 & 0.26 & 0.26 & 0.26 \\
Valine & 0.82 & 0.82 & 0.82 & 0.82 & 0.82 \\
Digestible Valine & 0.71 & 0.71 & 0.71 & 0.71 & 0.71 \\
\hline
\end{tabular}




\begin{tabular}{lccccc}
\hline Histidine & 0.43 & 0.43 & 0.43 & 0.43 & 0.43 \\
Digestible Histidine & 0.37 & 0.37 & 0.37 & 0.37 & 0.37 \\
Phenylalanine & 0.78 & 0.78 & 0.78 & 0.78 & 0.78 \\
Digestible Phenylalanine & 0.67 & 0.67 & 0.67 & 0.67 & 0.67 \\
Leucine & 1.44 & 1.44 & 1.44 & 1.44 & 1.44 \\
Digestible Leucine & 1.21 & 1.21 & 1.21 & 1.21 & 1.21 \\
Isoleucine & 0.66 & 0.66 & 0.66 & 0.66 & 0.66 \\
Digestible Isoleucine & 0.58 & 0.58 & 0.58 & 0.58 & 0.58 \\
\hline
\end{tabular}

Table 3. Body weight (gm) and other conformation traits (cm) in four varieties of indigenous Aseel chicken fed under different lysine regimens at the age of 6 weeks.

\begin{tabular}{|c|c|c|c|c|c|c|}
\hline \multicolumn{2}{|l|}{ Variables } & Body weight & $\begin{array}{l}\text { Shank } \\
\text { length }\end{array}$ & $\begin{array}{c}\text { Shank } \\
\text { circumference }\end{array}$ & $\begin{array}{l}\text { Drumstick } \\
\text { length }\end{array}$ & $\begin{array}{c}\text { Drumstick } \\
\text { Circumference }\end{array}$ \\
\hline \multicolumn{7}{|c|}{ Lysine Levels (\%)/Regimens (LR) } \\
\hline \multicolumn{2}{|c|}{$1.3(\mathrm{~L} 1)$} & $366.06 \pm 3.95^{\mathrm{c}}$ & $4.17 \pm 0.62^{\mathrm{c}}$ & $2.11 \pm 0.04^{\mathrm{b}}$ & $5.27 \pm 0.06^{\mathrm{c}}$ & $3.39 \pm 0.04^{\mathrm{b}}$ \\
\hline \multicolumn{2}{|c|}{$1.4-1.2(\mathrm{~L} 2)$} & $383.05 \pm 4.39^{b}$ & $4.37 \pm 0.08^{b}$ & $2.32 \pm 0.03^{\mathrm{a}}$ & $5.57 \pm 0.08^{b}$ & $3.61 \pm 0.03^{\mathrm{a}}$ \\
\hline \multicolumn{2}{|c|}{$1.5-1.3-1.1(\mathrm{~L} 3)$} & $409.59 \pm 4.62^{\mathrm{a}}$ & $4.58 \pm 0.06^{\mathrm{a}}$ & $2.32 \pm 0.03^{\mathrm{a}}$ & $5.78 \pm 0.06^{\mathrm{a}}$ & $3.60 \pm 0.03^{\mathrm{a}}$ \\
\hline \multicolumn{7}{|c|}{ Aseel Varieties (AV) } \\
\hline \multicolumn{2}{|c|}{ Lakha } & $384.57 \pm 6.00^{\mathrm{b}}$ & $4.63 \pm 0.06^{\mathrm{a}}$ & $2.29 \pm 0.04^{\mathrm{a}}$ & $5.73 \pm 0.07^{\mathrm{a}}$ & $3.56 \pm 0.04^{\mathrm{a}}$ \\
\hline \multicolumn{2}{|l|}{ Mianwali } & $383.47 \pm 6.07^{b}$ & $4.43 \pm 0.06^{\mathrm{b}}$ & $2.27 \pm 0.04^{\mathrm{a}}$ & $5.59 \pm 0.06^{\mathrm{a}}$ & $3.57 \pm 0.04^{\mathrm{a}}$ \\
\hline \multicolumn{2}{|l|}{ Mushki } & $402.23 \pm 3.88^{\mathrm{a}}$ & $4.43 \pm 0.91^{b}$ & $2.37 \pm 0.03^{\mathrm{a}}$ & $5.63 \pm 0.09^{\mathrm{a}}$ & $3.65 \pm 0.04^{\mathrm{a}}$ \\
\hline \multicolumn{2}{|c|}{ Peshawari } & $374.67 \pm 5.24^{\mathrm{b}}$ & $4.00 \pm 0.08^{c}$ & $2.07 \pm 0.04^{\mathrm{b}}$ & $5.20 \pm 0.08^{b}$ & $3.34 \pm 0.04^{\mathrm{b}}$ \\
\hline \multicolumn{7}{|c|}{ Lysine Levels $(\%) /$ Regimens × Aseel Varieties $(\mathrm{LR} \times \mathrm{AV})$} \\
\hline \multirow{4}{*}{$1.3(\mathrm{~L} 1)$} & Lakha & $356.25 \pm 7.19^{\mathrm{f}}$ & $4.46 \pm 0.08^{\mathrm{ab}}$ & $2.31 \pm 0.11^{\mathrm{bcd}}$ & $5.37 \pm 0.10^{\mathrm{cd}}$ & $3.56 \pm 0.11^{\mathrm{bcd}}$ \\
\hline & Mianwali & $352.00 \pm 8.34^{\mathrm{f}}$ & $4.33 \pm 0.10^{\mathrm{bc}}$ & $2.14 \pm 0.06^{\mathrm{de}}$ & $5.42 \pm 0.08^{\mathrm{cd}}$ & $3.44 \pm 0.06^{\mathrm{cd}}$ \\
\hline & Mushki & $385.70 \pm 6.36^{\text {cde }}$ & $4.25 \pm 0.10^{\mathrm{bc}}$ & $2.17 \pm 0.04^{\mathrm{de}}$ & $5.45 \pm 0.10^{\mathrm{bcd}}$ & $3.47 \pm 0.04^{\mathrm{cd}}$ \\
\hline & Peshawari & $370.30 \pm 7.81^{\mathrm{ef}}$ & $3.65 \pm 0.07^{\mathrm{d}}$ & $1.84 \pm 0.05^{\mathrm{f}}$ & $4.85 \pm 0.07^{\mathrm{e}}$ & $3.08 \pm 0.03^{\mathrm{e}}$ \\
\hline \multirow{4}{*}{$1.4-1.2(\mathrm{~L} 2)$} & Lakha & $375.90 \pm 8.98^{\mathrm{def}}$ & $4.62 \pm 0.12^{\mathrm{ab}}$ & $2.31 \pm 0.03^{\mathrm{bcd}}$ & $5.82 \pm 0.12^{\mathrm{ab}}$ & $3.61 \pm 0.03^{\mathrm{abc}}$ \\
\hline & Mianwali & $394.65 \pm 8.74^{\text {bcde }}$ & $4.51 \pm 0.1^{\mathrm{ab}}$ & $2.42 \pm 0.05^{\mathrm{acb}}$ & $5.71 \pm 0.10^{\mathrm{abc}}$ & $3.72 \pm 0.05^{\mathrm{ab}}$ \\
\hline & Mushki & $405.10 \pm 5.36^{\mathrm{abc}}$ & $4.31 \pm 0.21^{\mathrm{bc}}$ & $2.45 \pm 0.04^{\mathrm{ab}}$ & $5.51 \pm 0.21^{\mathrm{bcd}}$ & $3.72 \pm 0.06^{\mathrm{ab}}$ \\
\hline & Peshawari & $356.55 \pm 7.91^{\mathrm{f}}$ & $4.03 \pm 0.17^{\mathrm{c}}$ & $2.09 \pm 0.05^{\mathrm{e}}$ & $5.23 \pm 0.17^{\mathrm{d}}$ & $3.39 \pm 0.05^{\mathrm{d}}$ \\
\hline \multirow{4}{*}{$\begin{array}{l}1.5-1.3- \\
1.1(\mathrm{~L} 3)\end{array}$} & Lakha & $421.55 \pm 9.23^{\mathrm{a}}$ & $4.81 \pm 0.08^{\mathrm{a}}$ & $2.27 \pm 0.07^{\text {bcde }}$ & $6.01 \pm 0.08^{\mathrm{a}}$ & $3.53 \pm 0.07^{\mathrm{cd}}$ \\
\hline & Mianwali & $403.75 \pm 10.83^{\mathrm{abc}}$ & $4.47 \pm 0.11^{\mathrm{ab}}$ & $2.24 \pm 0.08^{\text {cde }}$ & $5.67 \pm 0.11^{\mathrm{abc}}$ & $3.54 \pm 0.08^{\mathrm{bcd}}$ \\
\hline & Mushki & $415.90 \pm 6.80^{\mathrm{ab}}$ & $4.72 \pm 0.11^{\mathrm{a}}$ & $2.50 \pm 0.04^{\mathrm{a}}$ & $5.92 \pm 0.11^{\mathrm{a}}$ & $3.77 \pm 0.05^{\mathrm{a}}$ \\
\hline & Peshawari & $397.15 \pm 9.32^{\mathrm{abcd}}$ & $4.32 \pm 0.09^{\mathrm{bc}}$ & $2.28 \pm 0.03^{\mathrm{bcd}}$ & $5.52 \pm 0.09^{\mathrm{bcd}}$ & $3.56 \pm 0.03^{\mathrm{bcd}}$ \\
\hline \multicolumn{3}{|c|}{ Source of Variation } & \multicolumn{4}{|c|}{$P$-value } \\
\hline \multicolumn{2}{|c|}{ LR } & $<.0001$ & $<.0001$ & $<.0001$ & $<.0001$ & $<.0001$ \\
\hline \multicolumn{2}{|l|}{ AV } & 0.0014 & $<.0001$ & $<.0001$ & $<.0001$ & $<.0001$ \\
\hline \multicolumn{2}{|l|}{$\mathrm{LR} \times \mathrm{AV}$} & $<.0001$ & $<.0001$ & 0.0004 & $<.0001$ & 0.0006 \\
\hline
\end{tabular}

Values and standard units of all parameters have been mentioned as Mean \pm SE, superscripted alphabets represent significant $(P \leq 0.05)$ variations, wherein $a>b>c>d \ldots$.

Table 4. Body conformation traits $(\mathrm{cm})$ in four varieties of indigenous Aseel chicken fed under different lysine regimens at the age of 6 weeks.

\begin{tabular}{lccccc}
\hline Variables & Body length & Keel length & Wing span & Wing spread & Breast width \\
\hline Lysine Levels (\%)/Regimens (LR) & & & & & \\
$1.3(\mathrm{~L} 1)$ & $28.95 \pm 0.15$ & $4.77 \pm 0.06^{\mathrm{c}}$ & $36.69 \pm 0.13^{\mathrm{ab}}$ & $17.65 \pm 0.14^{\mathrm{b}}$ & $1.13 \pm 0.02^{\mathrm{c}}$ \\
$1.4-1.2(\mathrm{~L} 2)$ & $29.40 \pm 0.20$ & $4.97 \pm 0.08^{\mathrm{b}}$ & $36.18 \pm 0.75^{\mathrm{b}}$ & $17.93 \pm 0.17^{\mathrm{b}}$ & $1.29 \pm 0.02^{\mathrm{b}}$ \\
$1.5-1.3-1.1$ (L3) & $29.39 \pm 0.20$ & $5.18 \pm 0.06^{\mathrm{a}}$ & $37.92 \pm 0.15^{\mathrm{a}}$ & $18.71 \pm 0.15^{\mathrm{a}}$ & $1.45 \pm 0.03^{\mathrm{a}}$ \\
Aseel Varieties (AV) & & & & \\
Lakha & $29.49 \pm 0.29^{\mathrm{ab}}$ & $5.23 \pm 0.06^{\mathrm{a}}$ & $36.59 \pm 1.02$ & $18.62 \pm 0.21^{\mathrm{a}}$ & $1.34 \pm 0.04^{\mathrm{a}}$ \\
Mianwali & $28.89 \pm 0.15^{\mathrm{b}}$ & $5.03 \pm 0.06^{\mathrm{b}}$ & $37.22 \pm 0.16$ & $18.12 \pm 0.16^{\mathrm{b}}$ & $1.28 \pm 0.03^{\mathrm{a}}$ \\
Mushki & $29.63 \pm 0.18^{\mathrm{a}}$ & $5.03 \pm 0.09^{\mathrm{b}}$ & $37.31 \pm 0.16$ & $18.28 \pm 0.17^{\mathrm{ab}}$ & $1.34 \pm 0.04^{\mathrm{a}}$ \\
\hline
\end{tabular}




\begin{tabular}{|c|c|c|c|c|c|c|}
\hline \multicolumn{2}{|l|}{ Peshawari } & $28.98 \pm 0.19^{\mathrm{b}}$ & $4.60 \pm 0.07^{\mathrm{c}}$ & $36.61 \pm 0.20$ & $17.37 \pm 0.17^{\mathrm{c}}$ & $1.20 \pm 0.03^{\mathrm{b}}$ \\
\hline \multicolumn{7}{|c|}{ Lysine Levels (\%)/Regimens × Aseel Varieties (LR × AV) } \\
\hline \multirow{4}{*}{$1.3(\mathrm{~L} 1)$} & Lakha & $29.48 \pm 0.37$ & $5.06 \pm 0.08^{\mathrm{ab}}$ & $36.79 \pm 0.27$ & $17.69 \pm 0.27^{\mathrm{cd}}$ & $1.19 \pm 0.03^{\mathrm{de}}$ \\
\hline & Mianwali & $28.19 \pm 0.16$ & $4.93 \pm 0.10^{\mathrm{bc}}$ & $36.69 \pm 0.25$ & $17.59 \pm 0.25^{\mathrm{cd}}$ & $1.16 \pm 0.05^{\mathrm{ef}}$ \\
\hline & Mushki & $29.09 \pm 0.22$ & $4.85 \pm 0.10^{\mathrm{bc}}$ & $36.70 \pm 0.17$ & $17.83 \pm 0.27^{\mathrm{cd}}$ & $1.14 \pm 0.02^{\mathrm{ef}}$ \\
\hline & Peshawari & $29.06 \pm 0.32$ & $4.25 \pm 0.07^{\mathrm{d}}$ & $36.59 \pm 0.36$ & $17.49 \pm 0.37^{\text {cd }}$ & $1.03 \pm 0.05^{\mathrm{f}}$ \\
\hline \multirow{4}{*}{$1.4-1.2(\mathrm{~L} 2)$} & Lakha & $29.45 \pm 0.53$ & $5.22 \pm 0.13^{\mathrm{ab}}$ & $34.46 \pm 3.01$ & $18.74 \pm 0.41^{\mathrm{ab}}$ & $1.29 \pm 0.02^{\mathrm{bcd}}$ \\
\hline & Mianwali & $29.17 \pm 0.26$ & $5.11 \pm 0.10^{\mathrm{ab}}$ & $37.01 \pm 0.25$ & $17.91 \pm 0.25^{\mathrm{cd}}$ & $1.26 \pm 0.04^{\text {cde }}$ \\
\hline & Mushki & $30.02 \pm 0.42$ & $4.91 \pm 0.21^{\mathrm{bc}}$ & $37.12 \pm 0.15$ & $18.02 \pm 0.15^{\mathrm{bc}}$ & $1.38 \pm 0.06^{\mathrm{abc}}$ \\
\hline & Peshawari & $28.98 \pm 0.30$ & $4.63 \pm 0.17^{\mathrm{c}}$ & $36.15 \pm 0.33$ & $17.05 \pm 0.33^{\mathrm{d}}$ & $1.23 \pm 0.04^{\text {cde }}$ \\
\hline \multirow{4}{*}{$1.5-1.3-1.1(\mathrm{~L} 3)$} & Lakha & $29.56 \pm 0.61$ & $5.41 \pm 0.08^{\mathrm{a}}$ & $38.53 \pm 0.18$ & $19.43 \pm 0.18^{\mathrm{a}}$ & $1.53 \pm 0.09^{\mathrm{a}}$ \\
\hline & Mianwali & $29.30 \pm 0.26$ & $5.07 \pm 0.11^{\mathrm{ab}}$ & $37.97 \pm 0.21$ & $18.87 \pm 0.21^{\mathrm{a}}$ & $1.43 \pm 0.05^{\mathrm{ab}}$ \\
\hline & Mushki & $29.78 \pm 0.23$ & $5.31 \pm 0.11^{\mathrm{a}}$ & $38.10 \pm 0.32$ & $19.00 \pm 0.32^{\mathrm{a}}$ & $1.51 \pm 0.08^{\mathrm{a}}$ \\
\hline & Peshawari & $28.91 \pm 0.37$ & $4.92 \pm 0.09^{\mathrm{bc}}$ & $37.10 \pm 0.33$ & $17.56 \pm 0.16^{\mathrm{cd}}$ & $1.33 \pm 0.02^{\mathrm{bcd}}$ \\
\hline \multicolumn{2}{|c|}{ Source of Variation } & & & $P$-value & & \\
\hline \multicolumn{2}{|c|}{ LR } & 0.1405 & $<.0001$ & 0.0224 & $<.0001$ & $<.0001$ \\
\hline \multicolumn{2}{|l|}{$\mathrm{AV}$} & 0.0282 & $<.0001$ & 0.6564 & $<.0001$ & 0.0013 \\
\hline \multicolumn{2}{|l|}{$\mathrm{LR} \times \mathrm{AV}$} & 0.5098 & $<.0001$ & 0.5143 & $<.0001$ & $<.0001$ \\
\hline
\end{tabular}

Values and standard units of all parameters have been mentioned as $\mathrm{M} \pm \mathrm{SE}$, superscripted alphabets represent significant $(P \leq 0.05)$ variations, wherein $\mathrm{a}>\mathrm{b}>\mathrm{c}>\mathrm{d}$

\section{DISCUSSION}

The present outcomes regarding BW, SL and DL are in close agreement with those of Batool et al. (2017c). According to Senapati et al. (2017), SL and KL were directly proportional to $\mathrm{BW}$ and increase in $\mathrm{BW}$ increased both SL and KL and his findings were also authenticated by some other researchers. They proved that frame size and skeletal growth of broiler breeders were primarily effected by BW and were highly influenced by the differences in dietary proteins and energy. Some other studies also reported the positive effect of lysine supplementation in low protein diets offered to growing broilers (Saima et al., 2010) and of BW on SL and KL growth structure of broiler strains (Yahaya et al., 2012). Lysine supplementation in cotton seed meal also had an ameliorating influence on BW in male Japanese quails (Ilyas et al., 2007). The similar findings of SC and DC had been reported by Batool et al. (2017c) in indigenous Aseel chicken fed with different dietary lysine regimens, wherein significantly $(P \leq 0.05)$ more developed SC and DC were noted in birds fed with L3 lysine regimen, while Lakha and Mushki showed the superiority in body conformation traits over other varieties of Aseel chicken. The current study also revealed KL and BWD significantly $(P \leq 0.05)$ largest in birds fed on L3 lysine regimen which are completely matched with those of Batool et al. (2017c), who reported the largest KL and BWD among Aseel chickens fed under three-phase feeding lysine regimen. Dosković et al. (2017) also demonstrated the effect of different dietary protein levels on body conformation traits of broiler chicken and found that when supplementation of dietary protein and protease levels were prolonged till $14^{\text {th }}$ day, absolute conformation traits including BW, SL, KL, breast depth (BD) and thigh girth (TG) were significantly $(P<0.05)$ increased except breast angle (BA) whose absolute measurement remained same even at different prolonged fattening periods. Blagojević (2011) also reported the same increase in SL of broilers fed with varying levels of proteins. Another study also demonstrated the positive effect of dietary lysine (low, medium and high) dense regimens on body conformations and concluded that medium dense lysine regimen (L2, with $1.30 \%$ lysine) was comparatively more better to improve the DL, DC, SL, SC, WSN, WSD, KL and BWD in Pakistani indigenous Aseel chickens (Hussain et al., 2018). The present verdicts concerning WSN and WSD are completely harmonized with those of Batool et al. (2017c), who also found the same effect of different lysine regimens on these body measurements and concluded that three phase feeding lysine regimen L3 $(1.5,1.3$ and $1.1 \%$ lysine) improved the WSN and WSD and other body conformation traits of native Aseel chicken. Some other studies have also proved the impact of protein and amino acid supplementation on body conformation characters and showed that bird's morphological features are strongly dependent upon nutrition, especially the protein and amino acid sources. Sikur et al. (2004) also endorsed his findings with the positive impact nutrient density with high protein and energy on BW, morphological development of breast muscles and overall growth of commercial Turkeys. Although, present findings showed a positive relationship between feeding regimens and body conformation traits, whilst the genetic and environmental impact also affect the body conformation traits among various strains, breeds and varieties (Das et al., 2014).

The findings of this study revealed that birds fed under lysine regimen L3 (three-phased) had significantly 
$(\mathrm{P} \leq 0.05)$ more developed body conformation traits, while Lakha and Mushki varieties of native Aseel chicken were more susceptible to retrieve the dietary impact on their growth potential.

Acknowledgement: The authors pay their gratitude to Prof. Dr. Muhammad Akram (late), Ex-Dean, Faculty of Animal Production and Technology, UVAS, Lahore for the planning of this research project and cooperation extended by the administration of UVAS, Pakistan to complete the present research work at ICGRC.

\section{REFERENCES}

Ahmad, Z., A.W. Sahota, M. Akram, A. Khalique, A.S. Jatoi, M. Shafique, M. Usman, and U. Khan (2014). Pre and post-moult productive efficiency in four varieties of indigenous Aseel chicken during different production cycles. J. Anim. Plant Sci. 24(5): 1276-1282.

Amao, S.R., L.O. Ojedapo, and A.O. Sosina (2010). Effect of strains on some growth traits of meattype chickens reared in derived savanna environment of Nigeria. J. Agric. Vet. Sci. 2(7): 58-64.

Babar, M.E., A. Nadeem, T. Hussain, A. Wajid, S.A. Shah, A. Iqbal, Z. Sarfraz, and M. Akram (2012). Microsatellite marker based genetic diversity among four varieties of Pakistani Aseel Chicken. Pakistan Vet. J. 32(2): 237-241.

Batool, T., A. Roohi, N. Roohi, and A. Mahmud (2017a). Impact of different dietary lysine regimens on blood biochemical profile and immune response in indigenous Aseel varieties. Pakistan Vet. J. 37(4): 393-398.

Batool, T., S. Farooq, N. Roohi, and A. Mahmud (2017b). Effect of different dietary lysine regimens on the behavioral response of indigenous Aseel varieties. Proc. UVAS, Lhr, Pakistan. p 41.

Batool, T., S. Farooq, N. Roohi, and A. Mahmud (2017c). Comparative evaluation of body conformation traits in four varieties of native Aseel chicken fed under different dietary lysine regimens. Proc. UVAS, Lhr, Pakistan. p 40.

Batool, T., N. Roohi, and A. Mahmud (2018). Subsequent effects of various dietary lysine regimens on production performance, egg persistency and economics of indigenous Aseel varieties. J. Anim. Plant Sci. 28(2): 396-406.

Blagojević, M. (2011). Influence of genotype on intensity of growth and quality of hull and meat broiler chickens in an extensive cultivation system. Ph.D. thesis. University of Novi Sad.

Chineke, C.A., B. Agaviezor, C.O.N. Ikeobi, and A.J. Ologun (2002). Some factors affecting body weight and measurements of rabbit at pre and post weaning ages. Proc. Nig. Soc. Anim. Prod., Nigeria. pp. 1-3.

Das, A.K., S. Kumar, A. Rahim, L.S. Kokatate, and A.K. Mishra (2014). Assessment of body conformation, feed efficiency and morphological characteristics in Rhode Island Red-white strain chicken. Ind. J. Anim. Sci. 84(9): 984-991.

Dohner, J.V. (2001). The encyclopedia of historic and endangered livestock and poultry breeds. Yale Agrarian Studies Series. Yale University Press. pp. 425-427.

Dosković, V., S. Bogosavljević-Bošković, Z. Škrbić, R. Đoković, S. Rakonjac, and V. Petričević (2017). Effect of dietary protein level and length of fattening period on dressing percentage and carcass conformation in broiler chickens. Biotech. Anim. Hus. 33(2): 211-219.

Hussain, J., I. Rabbani, S, Aslam, and H.A. Ahmad (2015). An overview of poultry industry in Pakistan. W. Poult. Sci. J. 71(4): 689-700.

Hussain, M., A. Mahmud, J. Hussain, S.N. Qaisrani, S. Ahmad, and A.U. Rehman (2018). Effect of dietary amino acid regimens on growth performance and body conformation and immune responses in Aseel chicken. Ind. J. Anim. Res. 30: 1-6.

Ilyas, M., M.K. Saleemi, F. Mahmood, and M.Z. Khan (2007). Pathological effects of feeding cottonseed meal with and without lysine in male Japanese quail (Coturnix japonica). Pakistan Vet. J. 27(2): 55-62.

Ogah, D.M. (2011). Assessing size and conformation of the body of Nigerian indigenous turkey. Slovak. J. Anim. Sci. 44(1): 21-27.

Paleja, H.I., F.P. Savalia, A.B. Patel, K. Khanna, P.H. Vataliya, and J.V. Solanki (2008). Genetic parameters in White Leghorn (IWN line) chicken. Ind. J. Poult. Sci. 43(2): 151-154.

Rehman, M. S., A. Mahmud, S. Mehmood, T.N. Pasha, M.T. Khan, and J. Hussain (2018). Animal wellbeing and behavior: Assessing behavior in Aseel pullets under free-range, part-time free-range and cage system during growing phase. Poult. Sci. 97(3): 725-732.

Rosario, M.F., M.A.N. Silva, A.A.D. Coelho, V.J.M. Savino, and C.T.S. Dias (2008). Canonical discriminant analysis applied to broiler chicken performance. Anim. Phy. 2(3): 419-424.

Saima, M. Z. U. Khan, M. A. Jabbar, A. Mehmud, M. M. Abbas, and A. Mahmood (2010). Effect of lysine supplementation in low protein diets on the performance of growing broilers. Pakistan Vet. J. 30(1): 17-20. 
SAS. 2002-03. SAS/State user's guide: Statistics. Version 9.1., SAS Institute Inc, Cary, North Carolina, USA.

Senapati, D.K., N.C. Behura, L. Samal, P.K. Mishra, S.K. Mishra, and B. Panigrahi (2017). Effects of Growth curves on Performance and Body Conformation traits in broiler breeder pullets. Adv. Biores. 8(6): 25-33.

Sikur, V.R., F.E. Robinson, D.R. Korver, R.A. Renema, and M.J. Zuidhof (2004). Effects of nutrient density on growth and carcass traits in fast-and slow-feathering female turkeys. Poult. Sci. 83(9): 1507-1517.

Singh, D.P. (2001). Aseel of India. In: Proceedings of a Seminar on Appropriate Poultry for Adverse Environment. pp. 96-100.

Sohail, A., A. Muhammad, J. Hussain, A. Iqbal, M. Usman, A. Rehman, and F. Hussnain (2013). Comparative study on productive performance, egg quality, egg geometry and hatching traits of three age groups of indigenous Peshawari Aseel chickens. Sci. J. Vet. Adv. 2(2): 21-25.
Udeh, I., and C.I. Ogbu (2011). Principal component analysis of body measurements in three strains of broiler chicken. Sci. W. J. 6(2): 11-14.

Yahaya, H.K., H. Ibrahim, and S. Abdusalam (2012). Correlation between body weight and body conformation of two broiler strains under the same dietary treatment. Int. J. Anim. Vet. Adv. 4(3): 181-183.

Yakubu, A., and G.L Mohammed (2012). Application of path analysis methodology in assessing the relationship between body weight and biometric traits of red Sokoto goats in northern Nigeria. Biotech. Anim. Hus. 28(1): 107-117.

Yunusa, A.J., and T.M. Adeoti (2014). Multivariate analysis for body weight and some linear body measurements of Nigerian indigenous chickens. Slovak. J. Anim. Sci. 47: 142-148.

Zia, M.W., A. Khalique, S. Naveed, and J. Hussain (2017). Studies on growth pattern of different body measurements in indigenous Aseel chicken fed with selenium supplemented diets. Ind. J. Anim. Res. 51(4): 679-686. 